Conclusions HPS across schools demonstrated gaps in knowledge surrounding both HPV and the HPV vaccine. Specifically students knew HPV causes cervical cancer; however, major knowledge gaps persist in the prevention of HPV infection. These areas represent high-yield opportunities for improvement within HPS education to ensure dissemination of knowledge regarding cancer prevention.

\section{EPV093/\#95 CLINICAL CHARACTERISTICS, TREATMENT RESPONSE AND PROGNOSIS OF LOCALLY ADVANCED ADENOCARCINOMA OF THE CERVIX, A LOCAL STUDY}

M Yu*. Jose R. Reyes Memorial Medical Center, Obstetrics and Gynecology, Manila, Philippines

\subsection{6/ijgc-2021-IGCS. 161}

Objectives The objective of this study is to determine the clinical characteristics, treatment response and prognosis of locally advanced adenocarcinoma of the cervix who underwent concurrent chemoradiotherapy with weekly Cisplatin or Carboplatin in comparison to squamous cell carcinoma.

Methods Outpatient charts of the cervical cancer patients from the outpatient department of Section of Gynecologic Oncology of a tertiary hospital were retrospectively reviewed.

Results Among the 979 charts reviewed, only 278 patients were included in the analysis. Seventy-five percent of the patients had squamous cell carcinoma and only 20\% had adenocarcinoma. Baseline characteristics were comparable. Ninety-eight percent had Cisplatin-based concurrent chemoradiotherapy. Median follow up was 17 months, with $75 \%$ of the patients had complete response and $16 \%$ had recurrent disease. Most common site of recurrence was cervix, lungs and bones. Disease free survival and overall survival was the same for adenocarcinoma and squamous cell carcinoma.

Conclusions Patients with locally advanced adenocarcinoma of the cervix who underwent concurrent chemoradiation had the same treatment response and prognosis to patients with squamous cell carcinoma.

\section{EPV093a/\#176 A MACHINE LEARNING APPROACH APPLIED TO GYNECOLOGICAL ULTRASOUND TO PREDICT PROGRESSION-FREE SURVIVAL IN OVARIAN CANCER PATIENTS}

${ }^{1}$ F Arezzo, ${ }^{2} \mathrm{~V}$ Loizzi, ${ }^{3} \mathrm{G}$ Cazzato, ${ }^{1} \mathrm{C}$ Lombardi, ${ }^{4} \mathrm{~V}$ Venerito, ${ }^{1} \mathrm{E}$ Cicinelli, ${ }^{1} \mathrm{G}$ Cormio ${ }^{*}$ ${ }^{1} \mathrm{CM}$ Santarsiero. ${ }^{1}$ Policlinico of Bary, Obstetrics and Gynecology Department, Bari, Italy; 2University of Bari 'Aldo Moro', Interdisciplinar Department of Medicine, Bari, Italy; ${ }^{3}$ Department of Emergency and Organ Transplantation, Pathology Section, Bari, Italy; ${ }^{4}$ Department of Emergency and Organ Transplantation, Rheumatology Unit, Bari, Italy

\subsection{6/ijgc-2021-IGCS.162}

Objectives Ultrasound(US) is a cheap, non-invasive and wellrecognized image modality for diagnosing and assessing ovarian cancer(OC). However, approximately $18 \%$ to $31 \%$ of adnexal lesions detected on US remain indeterminate. Machine learning(ML) is a promising tool for the implementation of complex multi-parametric algorithms. Despite the standardization of features capable of supporting the discrimination of ovarian masses into benign and malignant, there is the lack of accurate predictive modeling based on US examination for progression-free survival (PFS).

Methods In this retrospective observational study, we analyzed patients with epithelial ovarian cancer(EOC) who were followed in a tertiary center from 2018 to 2019. Demographic, clinical and laboratory characteristics were collected as well as information about post-surgery histopathology. Furthermore, we recorded data about US examinations according to International Ovarian Tumor Analysis(IOTA) classification. Proper feature selection was used to determine an attribute core set. Random Forest(RFF) algorithm was trained and validated with 10 -fold cross-validation to predict 12-month PFS. The accuracy of the algorithm was than assessed scoring accuracy and Area Under Receiver Operating Characteristic(AUROC).

Results Our analysis included n.32OC patients with mean age of $54.1 \pm 14.9$ years at diagnosis. Histotypes were n.19/32 (59.4\%) serous carcinoma, n.5/32(15.6\%) mucinous, n.5/32 (15.6\%) endometriod and n.3/32(9.4\%) clear cell. All patients underwent radical surgery. The attribute core set used to train machine learning algorithms is reported in figure 1. RFF showed an accuracy of 0.81 , AUROC 0.91 .

Conclusions We developed an accurate model to predict 12month PFS in patients with OC based on a ML algorithm applied to gynecological ultrasound evaluation, requiring few easy-to-collect attributes.

\section{EPV093b/\#769 PARADIGM SHIFT TO SENTINEL LYMPH NODE BIOPSY IN ENDOMETRIAL CANCER SURGERY: RECENT U.S. TRENDS}

${ }^{1}$ A Vallejo*, ${ }^{2} \mathrm{M}$ Klar, ${ }^{2} \mathrm{M}$ Hasanov, ${ }^{1} \mathrm{~K}$ Ciesielski, ${ }^{1} \mathrm{~L}$ Muderspach, ${ }^{1,3} \mathrm{~L}$ Roman, ${ }^{4} \mathrm{~J}$ Wright,

${ }^{1,3} \mathrm{~K}$ Matsuo. 'University of Southern California, Division Gynecology Oncology, Department of Obstetrics and Gynecology, Los Angeles, USA; ${ }^{2}$ University of Feibrug Faculty of Medicine, Obstetrics and Gynecology, Frieburg, Germany; ${ }^{3}$ University of Southern California, Norris Comprehensive Cancer Center, Los Angeles, USA; ${ }^{4}$ Columbia University College of PHysicians and Surgeons, Gynecologic Oncology, New York, USA

\subsection{6/ijgc-2021-IGCS. 163}

Objectives This study examined the population-level uptakes and outcomes of sentinel lymph node (SLN) biopsy for early endometrial cancer.

Methods This retrospective observational study queried the Surveillance, Epidemiology, and End Results Program, examining 83,139 women with endometrial cancer who underwent primary hysterectomy with lymph node evaluation for T1 disease from 2003-2018. Main outcome measures were (i) temporal trends and patient characteristics in utilization of SLN biopsy, and (ii) endometrial cancer-specific mortality associated with SLN biopsy.

Results The utilization of SLN biopsy increased significantly from $0.2 \%$ to $29.7 \%$ from $2005-2018$ with robust increase after $2016(\mathrm{P}<0.001$; top-panel). The uptake of SLN biopsy was higher for endometriod histology $(0.3 \%$ to $31.6 \%$ between 2005-2018) versus non-endometriod histology $(0.6 \%$ to $21.0 \%$ between $2006-2018$ )(both, $\mathrm{P}<0.001$ ). In a multivariable analysis, recent year surgery, endometrioid histology, well-differentiated tumors, T1a disease, and smaller tumor size remained independent characteristics for SLN biopsy use (all, $\mathrm{P}<0.05$ ). Performance of SLN biopsy was not associated with increased endometrial cancer-specific mortality compared to lymphadenectomy in endometrioid hystology (subdistribution- 

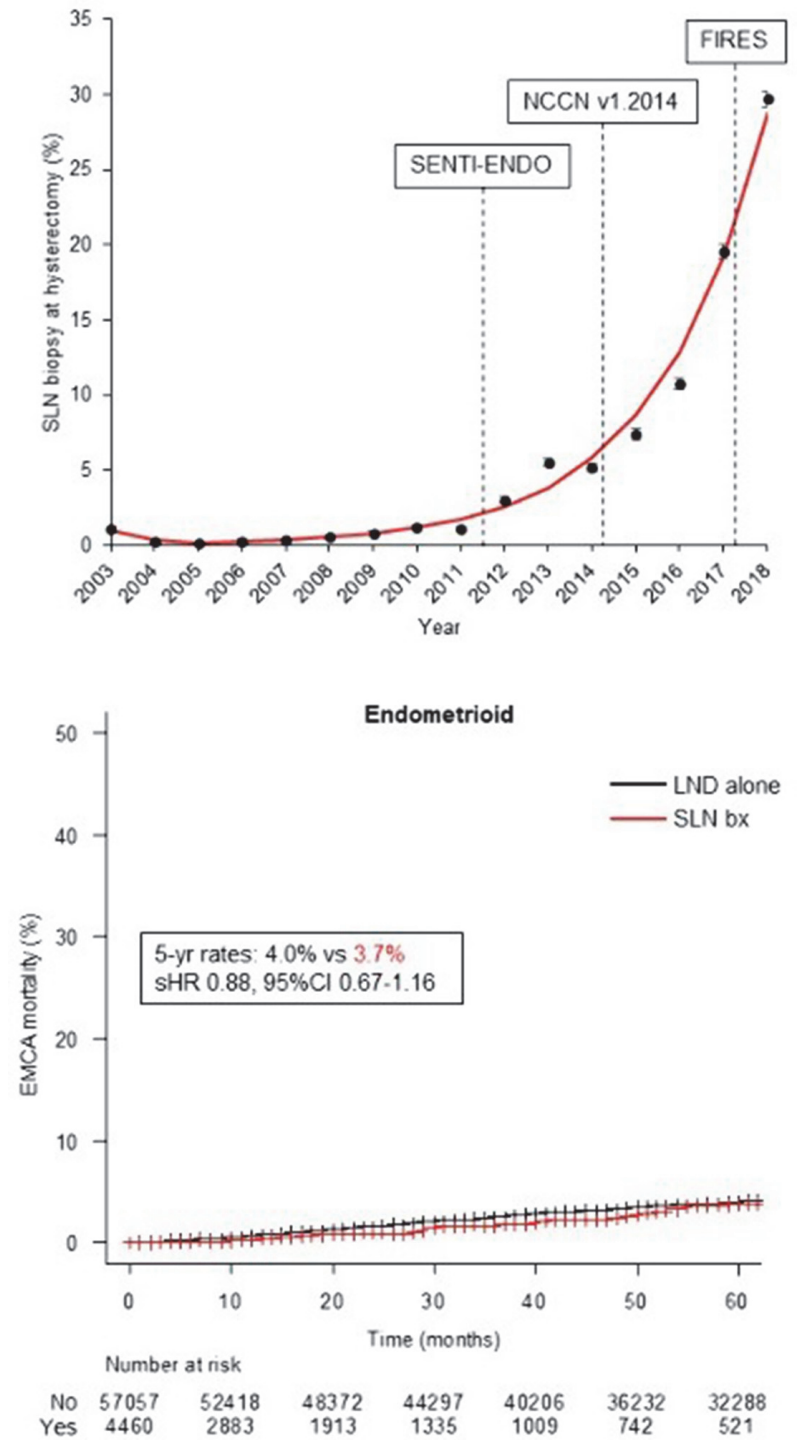

Abstract EPV093b/\#769 Figure 1

HR 0.96, 95\%CI 0.82-1.1; bottom-panel) and non-endometriod histology (subdistribution-HR 0.85 , 95\%CI 0.69-1.0) in propensity score weighted models. In low-risk endometrial cancer, the increase in recent SLN biopsy resulted in 15.3 percent point increase in the surgical nodal evaluation by 2018 (expected versus observed rates, 37.8\% versus 53.1\%).

Conclusions The landscape of surgical nodal evaluation is shifting from lymphadenectomy to SLN biopsy in early endometrial cancer. Effects of SLN biopsy-based surgical treatment on endometrial cancer survival warrants further confirmation.

\section{EPV094/\#103 MALIGNANT PERIVASCULAR EPITHELIOID TUMOR OF THE UTERUS ASSOCIATED WITH HYPERPROLACTINEMIA}

JI Argel*, D Benavides. Philippine General Hospital, Obstetrics and Gynecology, Manila, Philippines

10.1136/ijgc-2021-IGCS. 164
Objectives Malignant perivascular epitheliold tumors (PEComas) are rare mesenchymal tumors originating from perivascular epithelioid cells with specific histologic and immunologic features. Due to its rarity, lack of specific clinical findings, aggressive and unpredictable biologic behavior, this type of tumor is difficult to manage and there is no standard therapeutic strategy.

Methods A 34-year-old G1P1(1001) presented with a history of galactorrhea (elevated prolactin $313 \mathrm{ng} / \mathrm{mL}$ ) and irregular menstruation. On work-up, cranial magnetic resonance imaging (MRI) revealed no mass on the pituitary gland, abdominopelvic MRI showed a large uterine mass. She was initially treated medically which offered no relief of symptoms. She was then diagnosed and managed as a case of ectopic prolactin secreting leiomyoma uteri. Myomectomy was performed and prolactin level decreased to normal level $(6.3 \mathrm{ng} / \mathrm{mL})$ and with resolution of symptoms. Histopathology revealed malignant PEComa. Prolactin increased when tumor recurred and she underwent re-exploration and tumor debulking. Specimen from first and second operation were compared and shared the same histomorphological features. Immunohistochemical stain for prolactin was performed because of the suspicion of ectopic prolactin secreting tumor but revealed a negative result. The patient was given 3 cycles of Doxorubicin.

Results Endocrine paraneoplastic syndrome is the production of hormonal substances that produce unique clinical syndromes, example is prolactin. Ectopic prolactin secretion is the production of hormone by a cell type that does not normally produce the hormonal substance or produces it normally at very low levels.

Conclusions The index case showed malignant PEComa of the uterus associated with hyperprolactinemia with negative immunohistochemical stain for prolactin.

\section{EPV095/\#104 EXAMINING THE RISK OF COLORECTAL CANCER IN PATIENTS WITH MLH-1 PROMOTER HYPERMETHYLATED ENDOMETRIAL CANCER}

A Kanbergs*, L Philp, K James, T Randall. Massachusetts General Hospital, Obstetrics and Gynecology, Boston, USA

\subsection{6/ijgc-2021-IGCS. 165}

Objectives DNA Microsatellite instability (MSI) due to hypermethylation of the MLH1 gene leading to deficient DNA mismatch repair (MMR) is a frequent finding in sporadic endometrial (EC) and colorectal cancers (CRC). Individuals with germline MMR mutations have an $80 \%$ lifetime risk of colorectal cancer (CRC) and follow strict cancer screening protocols. It is unclear if women found to have sporadic MSI high EC have an increased risk of colorectal malignancy. The objective of this study was to determine if there is an increased risk of CRC in patients with MLH-1 promoter hypermethylated EC as compared to patients with microsatellite stable (MSS) disease.

Methods We performed a retrospective cohort study of all cases of EC with known MMR status treated at Massachusetts General Hospital between 2013-2019. Patients with germline MMR mutations were excluded. ICD-9/10 codes from electronic medical records were used to determine the incidence 